\title{
Continuous variable gate-model quantum computing
}

Mark Fingerhuth, Tomáš Babej, Peter Wittek

\section{Source}

Mark Fing erhuth, Tomáš Babej, Peter Wittek. (2018). Open source software in quantum

computing. PLOS ONE, vol. 13(12), e0208561.

The qubits are replaced by qumodes, which take continuous values. Conceptually this paradigm is closer to the physics way of thinking about quantum mechanics, and quantum optics in particular. Most of the language that describes these circuits uses the terminology of quantum optics. We will refer to this model as the continuous gate model. 